\title{
Comparison of uridine uptake at 24 hours with thymidine uptake at 72 hours in phytohaemagglutinin- stimulated cultures of pregnant and other subjects
}

\author{
T. E. BLECHER AND MILDRED J. THOMPSON
}

From the Department of Haematology, Nottingham General Hospital, Nottingham

SYNOPSIS As a possible more rapid test of lymphocyte reactivity to phytohaemagglutinin (PHA) than the standard estimation of thymidine uptake after 72 hours (T72), uridine uptake after 24 hours (U24) was studied in a total of 96 individuals. No reliable correlation between the two estimations was found in 29 outpatients showing a wide range of reactivity to PHA nor in 30 healthy control subjects nor in 16 pregnant subjects.

Conflicting reports have appeared in the literature as to whether lymphocyte reactivity to PHA is normal, depressed or enhanced in pregnancy. Our group of pregnant subjects showed a significant depression of both U24 (P < 0.01) and T72 (P < 0.02) results as compared to normal female controls.

Cultures of semipurified lymphocytes prepared by gradient centrifugation on a further 21 normal control subjects showed no better correlation between $U 24$ and T72 results than the above unpurifiedleucocyte cultures.

Transformation of lymphocytes by mitogens such as phytohaemagglutinin (PHA) is now well recognized as an index of an individual's cell-mediated immune competence (Valentine and Lawrence, 1971) and, more specifically, as a measure of T-lymphocyte responsiveness. This test has proved valuable in the assessment of immune deficiency and is becoming important in the management of neoplasia, for example, prognostication in acute leukaemia (Hersh et al, 1974).

PHA-induced transformation is normally assessed after three to five days' culture of whole-leucocyte or purified-lymphocyte suspensions, or even of whole blood, by the measurement of tritiated thymidine uptake. A test which could give an earlier measure of PHA responsiveness would have obvious clinical value. We have accordingly studied the uptake of tritiated uridine after only 24 hours of culture (U24) and compared this with the uptake of thymidine at 72 hours (T72) in 96 subjects. A close and consistent relationship between these two measurements would suggest that the shorter uridine estimation could reliably replace the standard 72-hour thymidine uptake determination where an early result was required clinically. We have been

Received for publication 13 January 1976 unable to find any previous clinical studies in which these two measurements have been systematically compared with this aim in mind, and have found very few reports of their combined estimation in patient studies.

With the intention of making the comparison over a wide range of states of lymphocyte responsiveness, samples were studied from 29 unselected outpatients attending for a wide variety of complaints. Thirty normal control subjects were similarly tested. Pregnant subjects provide a readily available source of lymphocytes which have been reported by some authors to show a reduced response to PHA in vitro (Purtilo et al, 1972) although reported by others to show an unimpaired response (Watkins, 1972) or even increased reactivity (Carr and Stites, 1972). We therefore also studied PHA-responsiveness by U24 and T72 uptakes in samples from 16 pregnant subjects.

The above cultures were performed by a wholeleucocyte-suspension culture technique based on that of Fitzgerald (1971, 1972). To control the validity of this technique we also examined the correlation between U24 and T72 uptakes obtained on cultures of semi-purified lymphocyte suspensions prepared by gradient centrifugation from a further 21 control subjects' samples. 


\section{Methods}

Twenty-millilitre samples of venous blood were obtained aseptically, with their consent, from $(a)$ as normal controls, 51 new nursing entrants, all female, aged 18-43 years; (b) 16 pregnant subjects, otherwise healthy, in the last trimester (aged 18-38 years); and (c) 29 unselected outpatients of both sexes referred to the laboratory by general practitioners and hospital clinics to have blood samples tested for a wide variety of complaints. Several were taking drugs of various sorts.

The samples were anticoagulated with $0.4 \mathrm{ml}$ of preservative-free heparin or, on a few occasions, defibrinated, which procedure we have found not to affect the results. After sedimentation of erythrocytes (Plasmagel) the supernatant leucocytes were washed thrice with medium T C 199 (Wellcome Reagents Ltd) containing $10 \%$ autologous plasma. The leucocytes were then diluted in this same medium to give a final concentration of $1 \times 10^{6}$ lymphocytes per ml (Fitzgerald, 1971). The outpatients', pregnant subjects', and 30 of the control subjects' samples were treated by the above method which will be referred to as the unpurified-leucocytes method. The remaining 21 control samples were subjected to gradient centrifugation in order to remove the great majority of the neutrophils from the lymphocytes. Thus, after red-cell sedimentation, the leucocytes were spun down, and resuspended in $5 \mathrm{ml}$ of $10 \%$ autologous plasma in medium 199, and this suspension was layered onto $2 \mathrm{ml}$ Ficoll-Hypaque (ratio 4:1, SG 1.078). After centrifugation at $400 \mathrm{~g}$ for 40 minutes the cells at the interface were removed by pipette, washed thrice, and resuspended to $1 \times$ $10^{6}$ lymphocytes per $\mathrm{ml}$ as above. These latter preparations will be referred to as semipurified lymphocytes. To $0.9 \mathrm{ml}$ aliquots of the cell suspensions were then added either $0.1 \mathrm{ml}$ of PHA (Wellcome, Reagent Grade, diluted 1:15), or, as controls, $0.1 \mathrm{ml}$ of medium 199 . They were then incubated in stoppered $5.0 \times 1.5 \mathrm{~cm}$ plastic tubes at $37^{\circ} \mathrm{C}$ in air. In these experiments PHA from three different batches was used. As this Reagent Grade is variable in mitogenic activity the results obtained with the different batches are treated separately in the Results section. On titration we found that a 1 in 15 dilution gave maximal mitogenic activity (T72); eg, results on Batch no $\mathrm{K} 9689$ were as follows:

\begin{tabular}{lll}
\hline$P H A$ dilution & Mean cpm $\left(\times 10^{-2}\right)$ & No of Cultures \\
\hline $1: 5$ & 38.0 & 11 \\
$1: 10$ & 44.0 & 12 \\
$1: 15$ & $51 \cdot 4$ & 11 \\
$1: 20$ & 39.8 & 12 \\
$1: 25$ & 44.6 & 11 \\
\hline
\end{tabular}

After 21 hours of culture $1 \mu \mathrm{Ci}$ of uridine $-5^{3} \mathrm{H}$ ( $5 \mathrm{Ci} / \mathrm{mmol}$, Amersham) was added to each of three of the PHA-containing tubes and three of the control tubes, and incubation was continued for three hours. Similarly, after 70 hours of culture $1 \mu \mathrm{Ci}$ of thymidine$-6^{3} \mathrm{H}$ ( $2 \mathrm{Ci} / \mathrm{mmol}$, Amersham) was added to three PHA-containing and three control tubes, and incubation was continued for two hours.

The cells were then washed twice in ice-cold saline and once in ice-cold $5 \%$ perchloric acid. After freezing and thawing, the deposits were again washed in the perchloric acid and solubilized in $0.5 \mathrm{ml}$ of Soluene 100 (Packard) at $40^{\circ} \mathrm{C}$ for one hour.

After neutralization with $1 \mathrm{M} \mathrm{HCl}$ the material was mixed with $8 \mathrm{ml}$ of a xylene-triton (2:1)-based scintillation fluid containing $5.5 \mathrm{~g} / 1$ of POP and $0.1 \mathrm{~g} / 1$ of POPOP, and the activity was measured in a Beckman LS-100 liquid scintillation counter. Correction for colour quenching was by the channelsratio method using an external standard. Background and tritium controls were always included.

The purpose of testing cultures at 24 and 72 hours not stimulated by PHA was to detect any patients who had pre-existing RNA- or DNA-synthesizing cells in their circulation. A few patients in whom the uridine uptake at 24 hours was $>2.0 \times 10^{3} \mathrm{cpm}$ were eliminated from the present series on this account. During the earlier part of the study constant aliquots $(\bumpeq 50 \mu \mathrm{l})$ were removed from each culture after completion of incubation, and smears were prepared and examined after staining. In all cases there was a very close correlation between blast cel counts and thymidine uptake.

\section{Results}

The results are shown as scattergrams in figures 1 to 4 . Each dot represents the mean counts per minute (cpm) of triplicate $1 \mathrm{ml}$ cultures. It is quite apparent

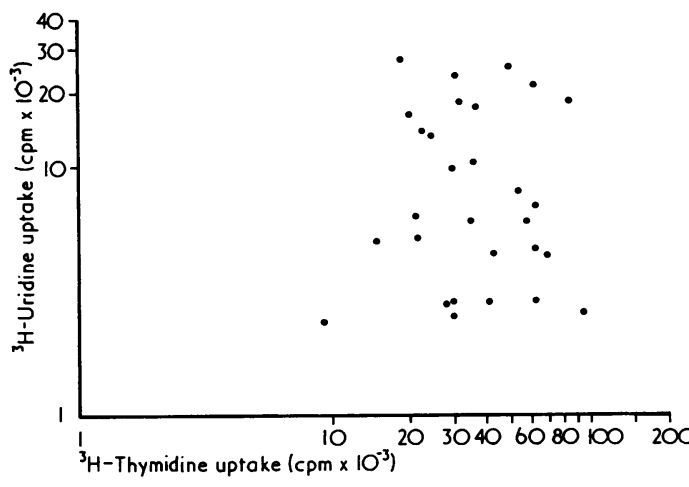

Fig 1 Comparison between U24 (ordinate) and T72 (abscissa) results of the 29 unselected outpatients. 
from the figures that there was no correlation between $U 24$ and $T 72$ results of either the unselected outpatients (fig 1), the controls (fig 2) or the pregnant subjects (fig 3). Coefficients of correlation between U24 and T72 results were as follows: (i) unselected outpatients (PHA batch no K6129): $r=0.08$; (ii) controls (a) 16 subjects using PHA batch no K6477 (solid dots on fig 2): $r=0.07$; (b) 14 subjects using PHA batch no K9689 (open circles on fig 2): $r=$ 0.34. The latter is not a significant correlation (for example, cf Documenta Geigy, 6th edition, p. 61); (iii) pregnant subjects (PHA batch no K9689): $\mathrm{r}=\mathbf{0} \cdot 17$.

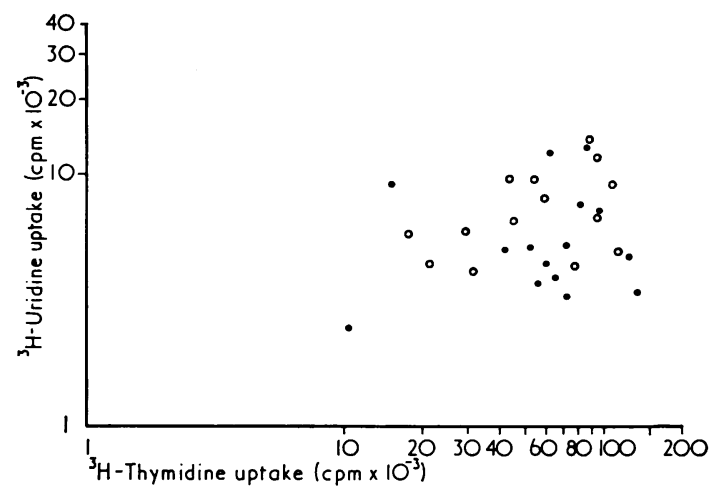

Fig 2 Comparison between U24 and T72 results of the 30 control subjects tested by the unpurified-leucocyte culture technique: 14 subjects tested with $P H A$ batch no K9689 shown as open circles; 16 subjects tested with PHA batch no K6477 shown as solid dots.

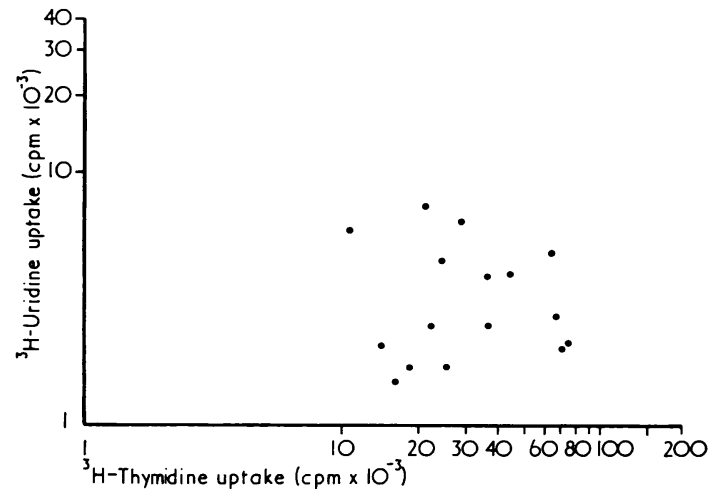

Fig 3 Comparison between $U 24$ and $T 72$ results of the 16 pregnant subjects.

Figure 4 shows that the semipurified-lymphocyte culture technique used on a further batch of 21 control subjects also gave no significant correlation between U24 and T72 estimations: $r=0.01$ (PHA batch no K6477).

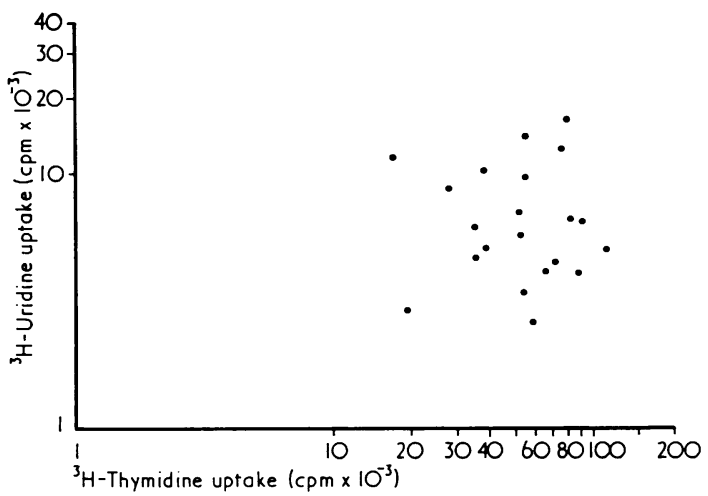

Fig 4 Comparison between $U 24$ and T72 results of the 21 control subjects tested by the semipurified-lymphocyte culture technique.

The PHA responsiveness of the 16 pregnant subjects' leucocytes was significantly less than that of the 14 female control subjects tested with the same batch of PHA (K9689) in terms of both U24 $(\mathrm{P}<0.01)$ and T72 $(\mathrm{P}<0.02)$ (Wilcoxon's Rank Sum Test).

\section{Discussion}

Although estimation of DNA synthesis after 72 hours' culture has become the standard method of assessing lymphocyte response to PHA, many other metabolic changes are known to precede the onset of DNA synthesis. Increased protein synthesis begins after three hours, and increased RNA synthesis and numerous other metabolic changes are detectable within the first hour of adding PHA (Peters and Hausen, 1971). Special techniques are required for the detection of some of these very early changes but the determination of uridine uptake requires only the facilities necessary for determination of thymidine uptake. We therefore chose to assess this estimation as a possible test which could easily be used by laboratories already using the thymidine estimation to replace the latter as a more rapid test of PHA responsiveness.

The results of the present study would appear to rule out the U24 estimation as a reliable substitute for the T72 estimation. There was no significant correlation between the U24 and T72 estimations in any of the groups.

Wide variability in the capacity of even normal individuals to respond to PHA is well known, as is the variability of the leucocyte culture technique itself (Yamamura, 1973). Hughes and Caspary (1970) concluded that because of technical vagaries and biological variation outside experimental 
control, significance should be attached only to major differences between cultures. We attempted to limit this variability by performing triplicate cultures and excluding contaminated cultures by morphological examination of stained slides.

We do not think that the variable numbers of neutrophils present in the unpurified-leucocyte cultures accounts for the lack of correlation between U24 and T72 estimations since removal of most of the neutrophils in the semipurified lymphocyte cultures still did not produce any significant correlation. Similarly Fitzgerald (1972) found no correlation between PHA response and non-lymphocyte cell content (between 30 and $70 \%$ ) of the preparation, and quotes similar findings by other workers.

The lack of correlation between the individual U24 and $T 72$ results might in part be due to the fact that the rate of uridine uptake is not necessarily strictly related to the rate of RNA synthesis (Kay and Handmaker, 1970). It also reflects the increased uridine kinase activity and enhanced transport of uridine across the cell membrane which are stimulated by PHA (Peters and Hausen, 1971). Like the phosphorylation of uridine, these may be ratelimiting factors in uridine uptake (Kay and Handmaker, 1970). If these factors varied widely in individual subjects' PHA-stimulated lymphocytes independently of later thymidine uptake, U24 and T72 values would not correlate well.

We have been unable to find in the literature any other systematic comparison of U24 and T72 estimations over a wide range of states of lymphocyte responsiveness in patients. In a small number of studies the results of both estimations are given but without calculation of the relationship between them. Tormey et al (1967) reported reduced RNA synthesis at 26 hours and DNA synthesis at 70 hours in PHA-stimulated leucocyte cultures from nine patients with agammaglobulinaemia compared to controls. Daniels et al (1970b) reported increased RNA and DNA synthesis in PHA-stimulated uraemic lymphocytes compared to normal lymphocytes. The same group (Daniels et al, 1970a) also reported increased mean RNA and DNA synthetic rates in both unstimulated and PHA-stimulated lymphocyte cultures from burned patients. Correlations between $\mathrm{U} 24$ and $T 72$ results were not calculated in these studies but it appears from their results that in each study discrepancies between the two estimations occurred.

More recently, in lymphocyte cultures from eight children with protein calorie malnutrition Burgess et al (1974) studied uridine uptake during the first 20 hours of culture and thymidine uptake over a similar period at the end of three days of culture. They noted that in the majority of cases when DNA synthesis was depressed the same was also true of RNA synthesis, but exceptions occurred. The correlation in this small group was found to be significant $\left(R_{8}=0.74, P<0.05\right)$.

Our finding of a significant depression of T72 in PHA-stimulated cultures from pregnant subjects as compared with normal controls confirms the findings of Purtilo et al (1972), Finn et al (1972), and Yu et al (1975). It should be mentioned that other workers have reported normal (Thiede et al, 1968; Watkins, 1972) or even enhanced lymphocyte reactivity to PHA in pregnancy (Carr and Stites, 1972). These conflicting results might in some way be accounted for by differences in culture techniques. The markedly depressed reactivity we and other workers have found appears to be due to an inhibitor in the maternal serum (Walker et al, 1972), possibly chorionic gonadotrophin which depresses lymphocyte PHA-reactivity (Kaye and Jones, 1971). It accords with other evidence of depressed cellular immunity in pregnancy which might account for the maternal tolerance of the antigenically foreign conceptus.

The present study extends these findings by demonstrating that uridine uptake at 24 hours is also very significantly depressed in PHA-stimulated leucocyte cultures from pregnant subjects in autologous plasma.

We should like to express our thanks to the Research Committee of the Trent Regional Health Authority for a grant, to the senior tutors of the Nottingham School of Nursing and the nurses and patients who kindly donated blood samples, and to Dr D. N. Brindley for advice on the scintillation techniques.

\section{References}

Burgess, B. J., Vos, G. H., Coovadia, H. M., Smythe, P. M., Parent, M. A., and Loening, W. E. L. (1974). Radioisotopic assessment of phytohaemagglutinin-stimulated lymphocytes from patients with protein calorie malnutrition. S. Afr. med. J., 48, 1870-1872.

Carr, M. C. and Stites, D. P. (1972). Reactivity of maternal lymphocytes to phytohaemagglutinin. (Letter.) Lancet, 1, 1073-1074.

Daniels, J. C., Cobb, E. K., Lynch, J. B., Lewis, S. R., Larson, D. L., and Ritzmann, S. E. (1970a). Altered nucleic acid synthesis in lymphocytes from patients with thermal burns. Surg. Gynec. Obstet., 130, 783-788.

Daniels, J. C., Sakai, H., Cobb, E. K., Remmers, A. R. Jr., Sarles, H. A., Fish, J. C., Levin, W. C., and Ritzmann, S. E. (1970b). Altered nucleic acid synthesis patterns in lymphocytes from patients with chronic uremia. Amer. $J$. med. Sci., 259, 214-227.

Finn, R., Hill, C. A. S., Govan, A. J., Ralfs, I. G., Gurney, F. J., and Denye, V. (1972). Immunological responses in pregnancy and survival of fetal homograft. Brit. med. J., 3, 150-152.

Fitzgerald, M. G. (1971). The establishment of a normal human population dose-response curve for lymphocytes 
cultured with PHA (phytohaemagglutinin). Clin. exp. Immunol., 8, 421-425.

Fitzgerald, M. G. (1972). A satisfactory quantitative test of lymphocyte response to phytohaemagglutinin for the definition of normal control values and recognition of immunological defects. J. clin. Path., 25, 163-168.

Hersh, E. M., Gutterman, J. U., Mavligit, G. M., McCredie, K. B., Burgess, M. A., Matthews, A., and Freireich, E. J. (1974). Serial studies of immunocompetence of patients undergoing chemotherapy for acute leukaemia. J. clin. Invest., 54, 401-408.

Hughes, D. and Caspary, E. A. (1970). Lymphocyte transformation in vitro measured by tritiated thymidine uptake. Int. Arch. Allergy, 37, 506-531.

Kay, J. E. and Handmaker, S. D. (1970). Uridine incorporation and RNA synthesis during stimulation of lymphocytes by PHA. Exp. Cell Res., 63, 411-421.

Kaye, M. D. and Jones, W. R. (1971). Effect of human chorionic gonadrotropin on in vitro lymphocyte transformation. Amer. J. Obstet. Gynec., 109, 1029-1031.

Peters, J. H. and Hausen, P. (1971). Effect of phytohaemagglutinin on lymphocyte membrane transport. 1. Stimulation of uridine uptake. Europ. J. Biochem., 19, 502-508.
Purtilo, D. T., Hallgren, H. M., and Yunis, E. J. (1972). Depressed maternal lymphocyte response to phytohaemagglutinin in human pregnancy. Lancet, 1, 769-771.

Thiede, H. A., Choate, J. W., and Dyre, S. (1968). Pregnancy and the lymphocyte. Amer. J. Obstet. Gynec., 102, 642-653.

Tormey, D. C., Kamin, R., and Fudenberg, H. H. (1967). Quantitative studies of phytohemagglutinin-induced DNA and RNA synthesis in normal and agammaglobulinemic leucocytes. J. exp. Med., 125, 863-872.

Valentine, F. T. and Lawrence, H. S. (1971). Cell-mediated immunity. Advanc. intern. Med., 17, $51-93$.

Walker, J. S., Freeman, C. B., and Harris, R. (1972). Lymphocyte reactivity in pregnancy (letter). Brit. med. J., 3, 469.

Watkins, S. M. (1972). Lymphocyte response to phytohaemagglutin in pregnancy. J. Obstet. Gynaec. Brit. Cwlth, 79, 990-993.

Yamamura, M. (1973). Standardisation of lymphocyte transformation to phytohaemagglutinin. Clin. exp. Immunol., 14, 457-467.

Yu, V. Y. H., Waller, C. A., MacLennan, I. C. M., and Baum, J. D. (1975). Lymphocyte reactivity in pregnant women and newborn infants. Brit. med. J., 1, 428-430. 